\title{
PortSmouth TAble Rotation Robotic technique for pan proctocolecotmy using DaVinci Si robotic system; P-STARR technique
}

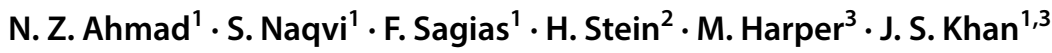

Received: 23 December 2017 / Accepted: 4 May 2018 / Published online: 8 June 2018

(c) The Author(s) 2018

\section{Introduction}

Robotic surgery offers a more natural feel and dexterity, a stable optical system and a tridimensional view of the operating field. Because of these technical benefits, robotic surgery has been widely accepted as a standard of care for urological surgery. However, the uptake of robotic colorectal surgery remains limited because of some inherent technical issues related to the setup and access of the target organs including challenges when operating in multiple quadrants of the abdomen.

The setup in robotic surgery is relatively fixed as compared to laparoscopic surgery. This rigid configuration of the robotic arms gives an excellent view of a target organ but is confined to a single abdominal quadrant. However, any access beyond the limits of a focused quadrant requires rearrangement of the robotic arms to complete the procedure effectively. This issue has been addressed for robotic rectal cancer surgery in several ways $[1,2]$. However, not much has been written on robotic assisted colon surgery.

One of the main challenges when considering a robotic surgical approach for proctocolectomy is achieving an optimal docking to access a large target organ in all four quadrants and the pelvis. A single docking of the robotic cart between the legs has previously been described [3]. Whilst that method may work well in selected cases, it limits access to the rectum. Similarly, a double docking requires extra time for complete reconfiguration of the robotic cart and theatre equipment. We present a novel and simple technique

J. S. Khan

mkhan702@aol.com

1 Queen Alexandra Hospital, Portsmouth Hsopitals NHS Trust, Portsmoth PO6 3LY, UK

2 Department of Clinical Development Engineering, Intuitive Surgical, Sunnyvale, CA, USA

3 School of Health Sciences and Social Work, University of Portsmouth, Portsmouth, UK of performing robotic proctocolectomy through a left sided docking (P-STARR technique).

\section{Pre-surgical positioning}

The patient is anaesthetised and positioned in a modified Lloyd Davies position on a vacuum bean bag mattress. The foot end of the operating table is then rotated $45^{\circ}$ clockwise so that it is positioned diagonally in the operating room, allowing more space to more around the table. The bony landmarks, pubic symphysis, xiphisternum, costal margins and both anterior superior iliac spines (ASIS) of the patient are marked to facilitate orientation. The midline of the patient is marked by joining the xiphisternum and pubic symphysis whilst the mid clavicular lines are drawn on both sides $8 \mathrm{~cm}$ lateral to the midline. Both ASIS are then joined to the midpoint of the midline and constitute spinoumbilical lines (Fig. 1). Pneumoperitoneum is created with a Veress needle in a pre-marked site for a robotic port (R2) in the left upper quadrant (Fig. 1).

\section{Port mapping}

The distance between two ports should be a minimum $8-10 \mathrm{~cm}$ to avoid collision of the robotic arms, with the optical port being positioned approximately $2 \mathrm{~cm}$ above and $2 \mathrm{~cm}$ to the right of the midpoint. Following this, the first robotic port (R1) is positioned at the intersection of right mid clavicular line and right spino-umbilical line the second robotic port (R2) is positioned approximately $8 \mathrm{~cm}$ below the left costal margin just medial to the mid clavicular line. The third robotic port (R3) is positioned $2 \mathrm{~cm}$ below the right costal margin medial to the mid clavicular line and finally, the assistant port is positioned midway between RI and R3 lateral to the mid clavicular line (Fig. 2). Once port mapping is achieved the patient should be re-positioned into 


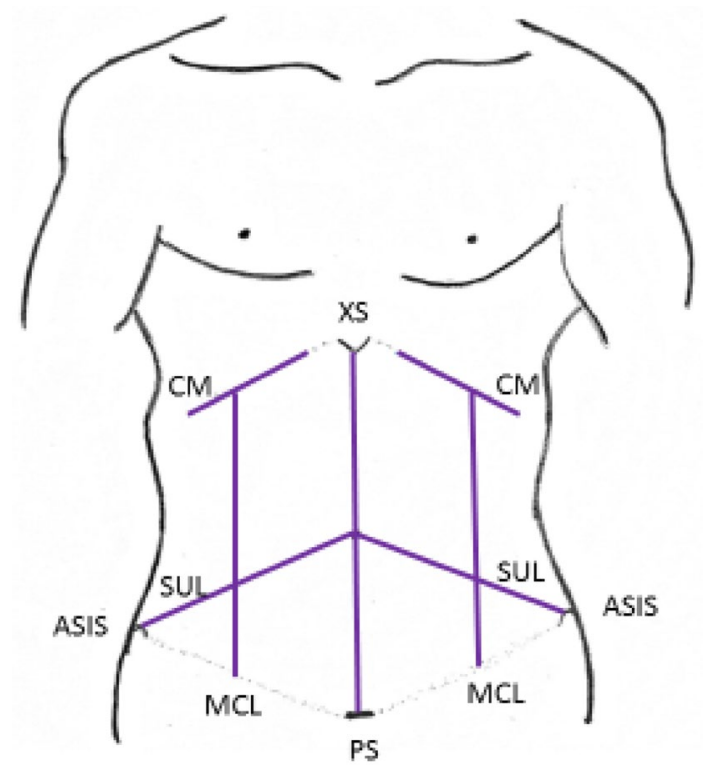

Fig. 1 Landmarks and model for port positioning. ASIS anterior superior iliac spine, $X S$ xiphisternum, $P S$ pubic symphysis, $M C L$ midclavicular line, $S U L$ spinoumbilical line, $C M$ costal margin

a $10^{\circ}$ Trendelenburg position with right lateral tilt in preparation for docking. The robotic cart is positioned and docked from the left side of the patient in line with the left spinoumbilical line. Once positioned satisfactorily, the robotic arms are connected to the ports and the robotic instruments are inserted under direct vision.

\section{The principle}

The usage of the different ports placed is dependent on the "stage" of the procedure. Docking and redocking of robotic arms to specific ports are part of this approach and will be described in detail in the following outlines on the different procedure "stages".

\section{Stage I}

The first part of the surgery involves ligation of inferior mesenteric vessels and mobilisation of the left colon and the splenic flexure. The set up described above has been reported previously [1] in a paper which details the procedure for robotic anterior resections and abdominoperineal excisions of the rectum (Fig. 3).

\section{Stage II}

The second stage of the procedure is the pelvic resection. For this, we follow the modified flip arm technique with the clockwise movement of R2 and R3. R2 is positioned more or less as a mirror image of R1 and R3 takes the position of R2 (Fig. 4). This set up is suitable for deep pelvic dissection where R2 is used for retraction in the pelvic cavity and dissection carried out with R1 and R3.

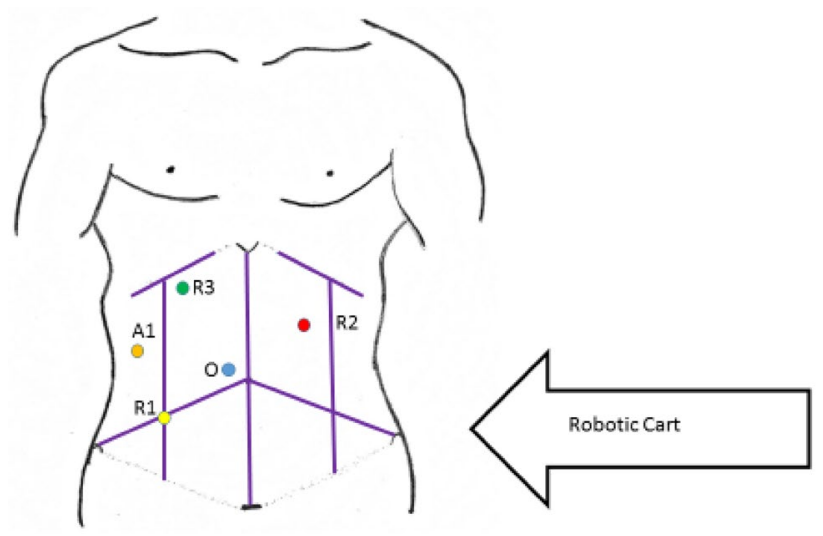

Fig. 3 Initial configuration of ports at the start of the procedure. $R 1$ first robotic port, $R 2$ second robotic port, $R 3$ third robotic port, $A 1$ assistant port for stage I and II, $O$ optical port
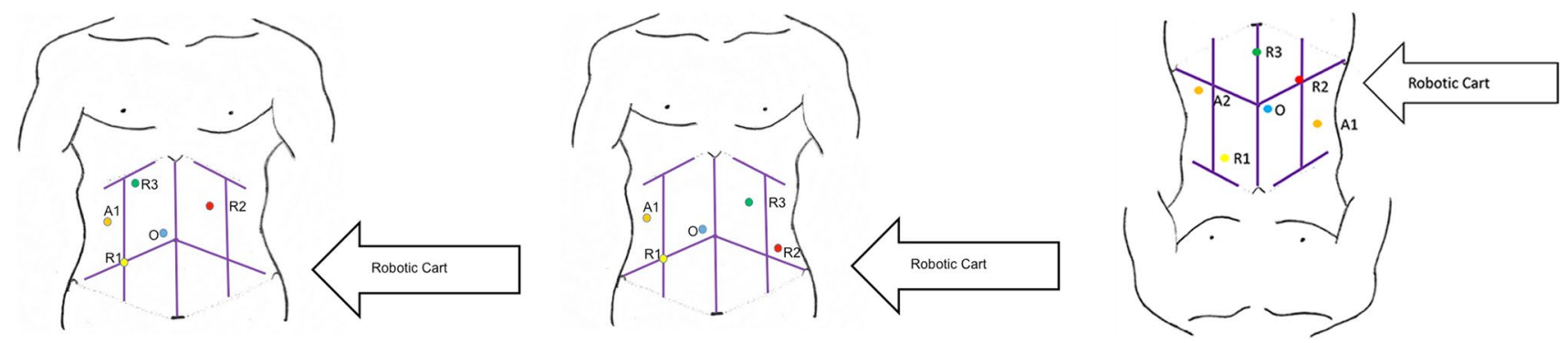

Fig. 2 Mapping and sequence of port positioning in the three stage procedure 


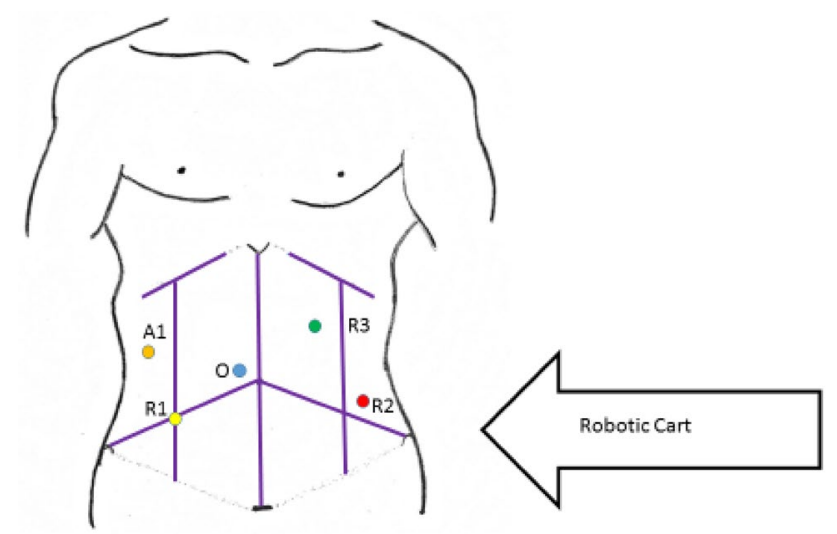

Fig. 4 Change of port positions for pelvic dissection. $R 1$ first robotic port, $R 2$ second robotic port, $R 3$ third robotic port, $A 1$ assistant port for stage I and II, $O$ optical port

\section{Stage III}

The right side of the colon is mobilised in stage III of the procedure. To complete this in single docking, the patient position is changed. The operating table is rotated $180^{\circ}$ clockwise along with the necessary anaesthetic equipment. The ports are moved again in a clockwise rotation. So, $\mathrm{R} 3$ is moved to the suprapubic area, R2 takes the position of $\mathrm{R} 1$ and $\mathrm{R} 1$ is moved to the position of R3. The previous $\mathrm{R} 2$ position is used for an assistant port on the right side of the colon. The clockwise movement of the ports in every step and the change of patient position for stage III is described in Fig. 5.

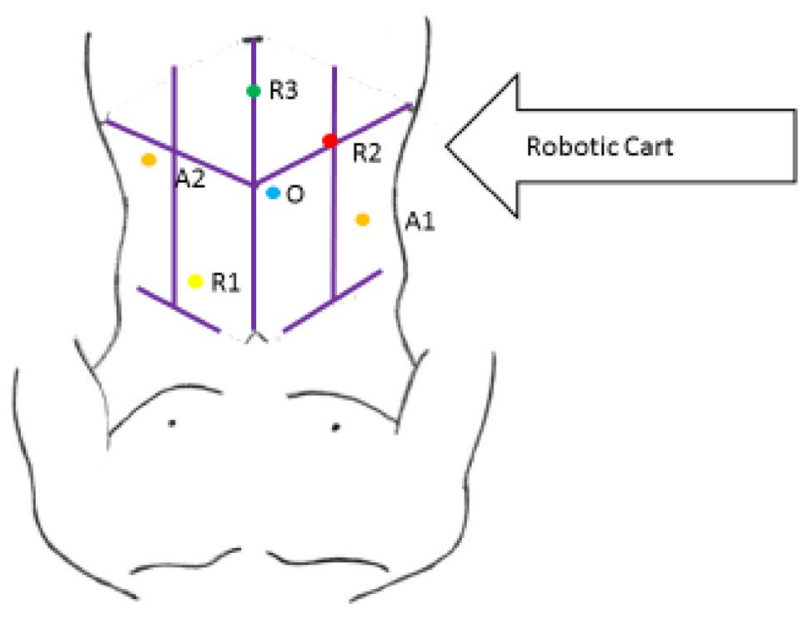

Fig. 5 The final configuration for right colectomy. $R 1$ first robotic port, $R 2$ second robotic port, $R 3$ third robotic port, $A 1$ assistant port for stage I and II, $A 2$ assistant port for stage III, $O$ optical port

\section{Preliminary evaluation of the P-STARR Technique}

The P-STARR technique has been sucessfully used in 8 patients who had robotic pan-proctocolectomy for ulcerative colitis. All procedures were carried out using DaVinci Si surgical system (Intuitive Surgical USA) The patient demographics and operative outcomes are described in Table 1. There was no significant technical difficulty faced during the surgery requiring redocking or repositioning of the arms beyond the procedure described above. Seven patients $(87.5 \%)$ had the end ileostomy fashioned in the right lower quadrant. One patient had an anterior resection in the past and requested ileostomy in the left lower quadrant. The specimens were extracted through a 4-5 cm extraction wound.

\section{Discussion}

An optimal setup with accurate port positioning to access the target organ is essential for an effective robotic procedure. Although the limitations faced by the Si system have been overcome to some extent by the newer version of $\mathrm{Da}$ Vinci $\mathrm{Xi}$, a process of mapping for precise port placements is still required [4]. This involves identification of anatomical

Table 1 Demographics and operative outcomes

\begin{tabular}{ll}
\hline Parameter & Mean (range) \\
\hline Total cases & 8 \\
Age, (years) & $50(24-79)$ \\
Gender & \\
Males:females & $6: 2$ \\
BMI & $23(20-25)$ \\
ASA & $2(1-3)$ \\
Blood loss (ml) & $14(0-50)$ \\
Operating time (min) & $300(180-375)$ \\
LOS (days) & $8(5-17)$ \\
Conversion & 0 \\
Technical clashing/ difficulty & Minimal, no \\
& extra ports, \\
$30-$ day mortality & repositioning \\
Complications & 0 \\
& 1 pelvic collec- \\
& tion, required \\
& radiological \\
drainage & 2 readmissions \\
Stoma reversed & with high \\
& stoma output \\
& 7 \\
\hline
\end{tabular}

$B M I$ body mass index, ASA American Society of Anesthesiologists, $L O S$ length of stay 
landmarks, and measurement of the distance between the ports and the target organs. The concept of mapping facilitates proper port positioning, and also permits development of a system for better configuration of the robotic arms for improved access to the target organ.

Docking is a process of joining the robotic arms to the trocars already inserted in the marked areas for access to the target organs. Any detachment of the robotic arms, subsequent movement of the robotic cart and reattachment of the arms to the ports constitutes a second docking. According to some studies, any movement of either the robotic cart or the patient which requires detaching and reattaching the robotic arms from different angles should be considered a second docking [4]. We believe in the model of a static robotic cart and favour movement of the patient table instead to improve the access to target organs when necessary. This would essentially be called a single position access as there has been no movement of the robotic cart.

Often, the difficulty in robotic colorectal surgery is a large target organ requiring a change of focus from one quadrant to another during the procedure, which may require redocking. Multi-quadrant access has been tried for anterior resection in a different way; by undocking one of the robotic arms during splenic flexure mobilisation and redocking for rectal dissection [5]. The flip arm technique basically utilizes an additional technical step of changing the position of two robotic arms for pelvic dissection [6]. The techniques reported for anterior resection lack the effectiveness required for a pan-proctocolectomy. This is because of a large surgical field and the need to access all four quadrants of the abdomen and pelvis. Our technique of left sided docking and a clockwise rotation provides an adequate access for safe completion of the procedure.

The time required for docking of the robotic cart has been implicated as a source of longer operating time. Dual docking will further increase this time. Manoeuvring a robotic cart inside theatre to dock from the other side adds more complexity and time to the task. We have found the use of table rotation in this setting very helpful. A standard Maquet Magnus table (MAQUET Holding B.V. \& Co. Germany) is rotated through $180^{\circ}$ at the end of stage II of the procedure. For patient safety the instruments are removed, the surgeon and the scrub team stay clean and the theatre team helps to rotate the table through $180^{\circ}$. The anaesthetist already prepared for this step using longer extension on the anaesthetic circuit and intravenous lines. The average time taken for this step has been $6(5-10)$ min. In our limited experience with this technique we have not come across any complications related to table rotation.

Our experience with this setup, although limited, has been very rewarding, with no difficulty in accessing the target organs, acceptable operating time and no major collisions of robotic arms during surgery. A clockwise reconfiguration of the setup takes place at every stage of the procedure making this a fairly simple technique to facilitate robotic proctocolectomy.

\section{Compliance with ethical standards}

Conflict of interest The authors declare that they have no conflict of interest.

Ethical approval The research was carreid out with the ethical standars ofd the institutional research and ethics committee and with the 1964 Helsinki declaration and its later amendments.

Informed consent Informed consent was obtained from all individual participants.

Open Access This article is distributed under the terms of the Creative Commons Attribution 4.0 International License (http://creativeco mmons.org/licenses/by/4.0/), which permits unrestricted use, distribution, and reproduction in any medium, provided you give appropriate credit to the original author(s) and the source, provide a link to the Creative Commons license, and indicate if changes were made.

\section{References}

1. Ahmed J, Nasir M, Flashman K, Khan J, Parvaiz A (2016) Totally robotic rectal resection: an experience of the first 100 consecutive cases. Int J Colorectal Dis 31(4):869-876

2. Toh JWT, Zakaria A, Yang I, Kim SH (2017) Totally robotic single docking low anterior resection for rectal cancer: pearls and pitfalls. Tech Coloproctol 21:893. https://doi.org/10.1007/s1015 1-017-1709-6

3. Roviello F, Piagnerelli R, Ferrara F, Scheiterle M, De Franco L, Marrelli D (2015) Robotic single docking total colectomy for ulcerative colitis: first experience with a novel technique. Int J Surg 21:63-67

4. Ngu JC, Sim S, Yusof S, Ng CY, Wong AS (2017) Insight into the da Vinci ${ }^{\circledR} \mathrm{Xi}$-technical notes for single-docking left-sided colorectal procedures. Int J Med Robot. https://doi.org/10.1002/ rcs. 1798

5. Uffort EE, Jensen JC (2011) Side docking the robot for robotic laparoscopic radical prostatectomy. JSLS 15(2):200-202

6. Obias V, Sanchez C, Nam A, Montenegro G, Makhoul R (2011) Totally robotic single-position 'flip' arm technique for splenic flexure mobilizations and low anterior resections. Int J Med Robot Comput Assist Surg 7(2):123-126 